\title{
Spectrum Sensing using AMC and TFT
}

\author{
M. Venkata Subbarao, P. Samundiswary
}

\begin{abstract}
Spectrum Sensing (SS) is a foremost step to implement next generation Cognitive Radio (CR) systems. The primary goal of a SS technique is to examine whether the Primary User (PU) is in active state or not by analyzing the surrounding radio environment. Traditional methods such as energy detection and Matched Filter Detection (MFD) schemes along with decision making circuits are generally used in SS. However, these techniques are developed under cooperative scenarios and they are used to sense single PU (narrowband sensing). In non-cooperative scenarios and fading channel conditions, traditional techniques produce higher false alarm. If Secondary User (SU) is occupied in the channel then SS task is more difficult. In order to overcome these limitations, a narrowband and wideband SS algorithm using Automatic Modulation Classification (AMC) and Time-Frequency Transform (TFT) is developed in this paper. The performance analysis of proposed AMC and TFT based SS technique under various channel conditions which is also described in this paper.
\end{abstract}

Keywords: AWGN, Fading Channels, FSWT, CR, SDR

\section{INTRODUCTION}

Based on the spectrum band of interest, the SS methods can be categorized in to Narrowband SS (NSS) and Wideband SS (WSS) techniques. The existing NSS approaches such as energy detection, MFD and feature based techniques is used to find whether a particular carrier of the spectrum is present in that geo-graphical location or not [1-3]. On the contrary, existing WSS [4-6] approaches such as FFT, WT and Filter bank techniques are used to identify group of carriers of a wideband to be occupied or vacant [7-8]. In fact, both these techniques are necessary for a successful SU communication. Initially, a WSS is required to identify the available/ unoccupied carriers in a specific spectrum band. After analyzing the available frequencies, best available band is selected according to application requirement so that SU can occupy that band. Once carrier frequency has been chosen, then SU initiates the data transfer. It is necessary to monitor the selected narrowband continuously, because to identify the arrival of PU [9-11]. In order to monitor the selected narrowband, a NSS is required. Based on these requirements, many researchers have developed different algorithms for NSS and WSS [12]. There are three major limitations that are identified from the literature which are faced by traditional NSS and WSS techniques and they are

Revised Manuscript Received on December 30, 2019.

* Correspondence Author

M. Venkata Subbarao, Department of ECE, Shri Vishnu Engineering College for Women, A.P, India. Email: mandava.decs@gmail.com

P. Samundiswary, Department of EE, School of Engg. \& Tech., Pondicherry University, India. Email: samundiswary_pdy@yahoo.com

(C) The Authors. Published by Blue Eyes Intelligence Engineering and Sciences Publication (BEIESP). This is an open access article under the CC BY-NC-ND license (http://creativecommons.org/licenses/by-nc-nd/4.0/) i. Most of the NSS techniques are developed under cooperative scenarios. If the information/ characteristics of the PU are unavailable, then these techniques are ineffective because of unknown threshold values for decision making.

ii. All traditional NSS techniques are unaware of SU signal characteristics. When a SU is occupied in a band these approaches are failed to identify the presence of the SU user.

iii. The WSS methods provide poor performance in fading channel conditions and they suffer with the more system complexity.

From these motivations, a new algorithm is developed in this research for narrowband \& wideband spectrum sensing using AMC and TFT.

\section{PROPOSED SPECTRUM SENSING TECHNIQUES}

In the proposed approach of AMC and TFT based SS, TFTs are used for WSS and AMC is used for the NSS. TFTs are capable of tracking the amplitude and frequency changes with respect to time. With the help of joint TFD it is possible to visualize entire wideband spectrum whether the narrowband are vacant or busy. The block diagram of WSS using TFTs as shown in Fig. 1. Initially, the signal is preprocessed for eliminating the out band noise then TFT scalogram is applied for extracting the joint Time-Frequency information. For WSS, proposed MST and MFSWT [13] are used as TFT for extracting the spectrum holes.

Let $r(t)$ is the received wideband signal. Then the MST of a signal $r(t)$ is given by [14-15]

$$
S(f, \tau)=\int_{-\infty}^{\infty} r(t) g(t-\tau) e^{-i 2 \pi f t} d t
$$

where $g(t)$ is a scalable gaussian window and it is given by

$$
g(t, f)=\frac{l+m \sqrt{|f|}}{K \sqrt{2 \pi}} e^{-\frac{(l+m \sqrt{f})^{2} t^{2}}{2 K^{2}}}, K>0
$$

where $K, l$ and $m$ are positive integers, $f$ is signal fundamental frequency

The received signal $r(t)$ includes PU signal $s(t)$ and noise $n(t)$ introduced in the signal due to either AWGN or fading conditions. The received signal $r(t)$ at the $\mathrm{SU}$ is given by

$$
r(t)=s(t)+n(t)
$$

The Energy Density (ED) is extracted from the TFT output for detecting presence of PU in the channel. The ED of the received signal using TFT is obtained as

$$
E(t, f)=|S(f, \tau)|^{2}
$$


Received

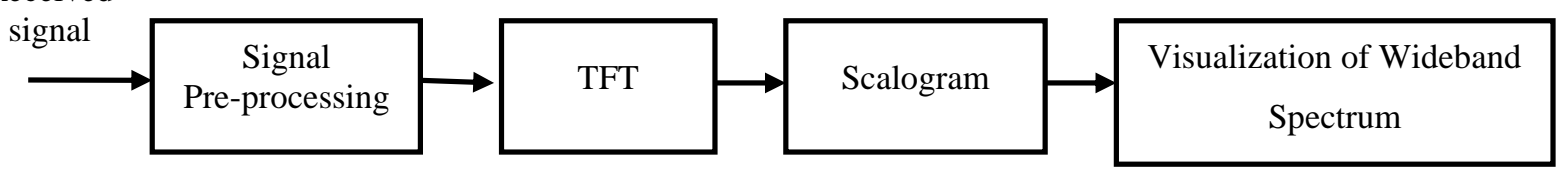

Fig. 1. Wideband Sensing with Proposed TFT

$$
E(t, f)=\left|\int r(t) g(t-\tau) e^{-i 2 \pi f t} d t\right|^{2}
$$

The received signal about $k^{\text {th }}$ narrowband PU is

$$
n_{k}(t)= \begin{cases}n_{k}(t) & k^{\text {th }} P U \text { is absen } \\ s_{k}(t)+n_{k}(t) & k^{\text {th } P U \text { is prese }}\end{cases}
$$

Therefore, the ED of $r_{k}(t)$ for the absence of PU and presence of PU are given by

$$
\begin{aligned}
E_{0}(t, f) & =\left|\int n_{k}(t) g(t-\tau) e^{-i 2 \pi f t} d t\right|^{2} \\
E(t, f) & =\left|\left(s_{k}(t)+n_{k}(t)\right) g(t-\tau) e^{-i 2 \pi f t} d t\right|^{2}
\end{aligned}
$$

$$
E(t, f)=\left|s_{k}(t) g(t-\tau) e^{-i 2 \pi f t} d t\right|^{2}+E_{0}(t, f)
$$

(9)

where $E_{0}(t, f)$ and $E_{1}(t, f)$ are ED of $k^{\text {th }}$ user if absent and present respectively.

The SS problem can be formulated with a binary hypotheses test as

$$
\left\{\begin{array}{ll}
H_{0}: & E(t, f)<T \\
H_{1}: & E(t, f) \geq T
\end{array} \quad \forall T>E_{0}(t, j)\right.
$$

where $H_{0}$ and $H_{1}$ are the hypothesis of PU absent and present respectively.

All traditional methods are unable to differentiate the occurrence of either PU or SU in a busy spectrum band. In order to differentiate PU with SU, dissimilar sets of modulation formats are allotted to PUs and SUs. The PUs use set $\{\mathrm{MP}\}$ modulation formats. All SUs uses different set of modulation formats $\{\mathrm{MS}\}$ for their communication. The NSS is done by checking the modulation format of the narrowband signal with the use of AMC schemes, for decision making. All traditional NSS algorithms produce binary decision whether the user is present or absent. But, with the use of AMC the proposed approach produces three outputs and they are, spectrum band is vacant, PU is present and SU is present.
In this method, first the narrowband signal is selected through the proper filtering and then the signal is passed through a modulation recognition device which produces the modulation format $\mathrm{M}_{\mathrm{i}}$ of the received signal. Further, the decision making device compares the modulation format of the received signal with the known sets of $\{\mathrm{MP}\}$ and $\{\mathrm{MS}\}$. If modulation type is not matched with $\{\mathrm{MP}\}$ and $\{\mathrm{MS}\}$, then it is identified as vacant i.e. no signal is present in that band. If it $\mathrm{M}_{\mathrm{i}}$ matches with $\{\mathrm{MP}$ \}, then the decision is $\mathrm{PU}$ is present else SU is present. The model of narrowband sensing with AMC is shown in Fig. 2.

\section{RESULTS \& DISCUSSIONS}

To illustrate the performance of TFT and AMC approach for SS, a wideband signal with four different narrowband frequencies and five active parts is considered for the simulation. The frequency components and active parts information of the wideband signal are shown in Table 1 and Table 2. To construct wideband signal, four different frequencies are considered which are used in TV broadcasting.

Table 1 Users Information

\begin{tabular}{|c|c|c|}
\hline Sl. No. & User & $\begin{array}{c}\text { Narrowband Central } \\
\text { Frequency } \\
\text { (MHz) }\end{array}$ \\
\hline 1 & $\mathrm{U}_{1}$ & 150 \\
\hline 2 & $\mathrm{U}_{2}$ & 250 \\
\hline 3 & $\mathrm{U}_{3}$ & 350 \\
\hline 4 & $\mathrm{U}_{4}$ & 450 \\
\hline
\end{tabular}

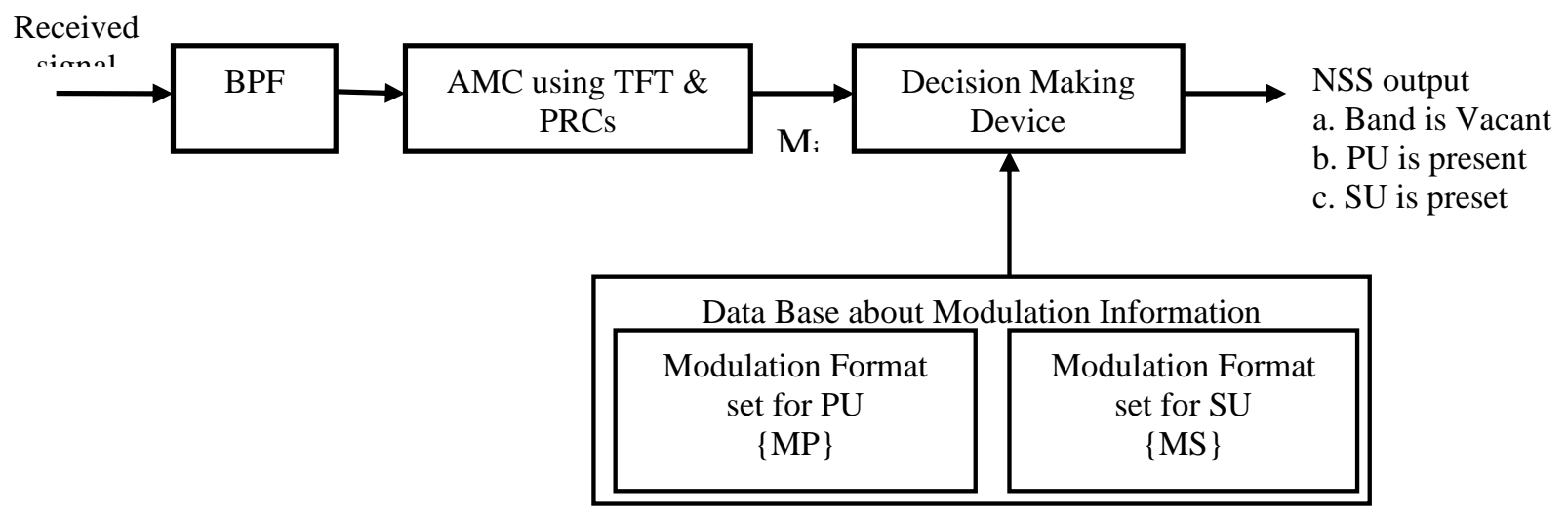

Fig. 2. Narrowband Sensing with AMC 
Table 2 Model of wideband signal

\begin{tabular}{|c|c|c|}
\hline Sl. No. & Active Part & Users Present \\
\hline 1 & $\mathrm{~A}_{1}$ & $\mathrm{U}_{1}, \mathrm{U}_{2}, \mathrm{U}_{3} \& \mathrm{U}_{4}$ \\
\hline 2 & $\mathrm{~A}_{2}$ & $\mathrm{U}_{2}, \mathrm{U}_{4}$ \\
\hline 3 & $\mathrm{~A}_{3}$ & $\mathrm{U}_{1}, \mathrm{U}_{3}$ \\
\hline 4 & $\mathrm{~A}_{4}$ & $\mathrm{U}_{2}, \mathrm{U}_{3} \& \mathrm{U}_{4}$ \\
\hline 5 & $\mathrm{~A}_{5}$ & $\mathrm{U}_{1}, \mathrm{U}_{2}, \& \mathrm{U}_{4}$ \\
\hline
\end{tabular}

The wideband SS with proposed MST is illustrated in Fig. 3. It is observed from Fig. 3, about the presence of user as well as absence of user. In A1, all PUs are present so that there is no free channel for SUs. Whereas in the second active part, first and third users are present, and second, fourth users are absent. The spectrum holes are represented with black dots in each active part. Similarly, the spectrum holes in all other active parts are clearly identified using the proposed TFTs.
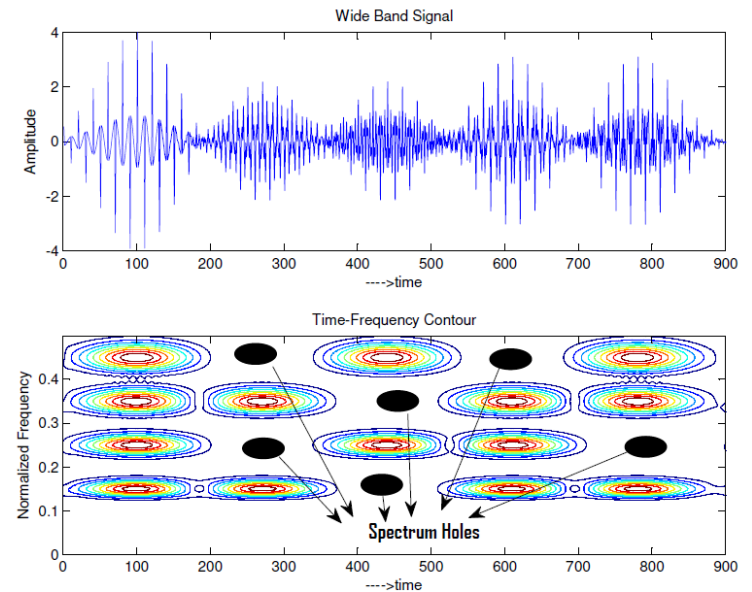

Fig. 3 Wideband SS with proposed TFT under ideal channel

Scalogram plot of the wideband signal using proposed TFT is illustrated in Fig. 4. For better detection and localization of the PUs, the scalogram is obtained from the absolute values of MST. From the Fig. 4, it is depicted that how the PUs are bruptly changes their presence in the channel. The frequency information of PUs at each active part is clearly displayed in the scalogram.

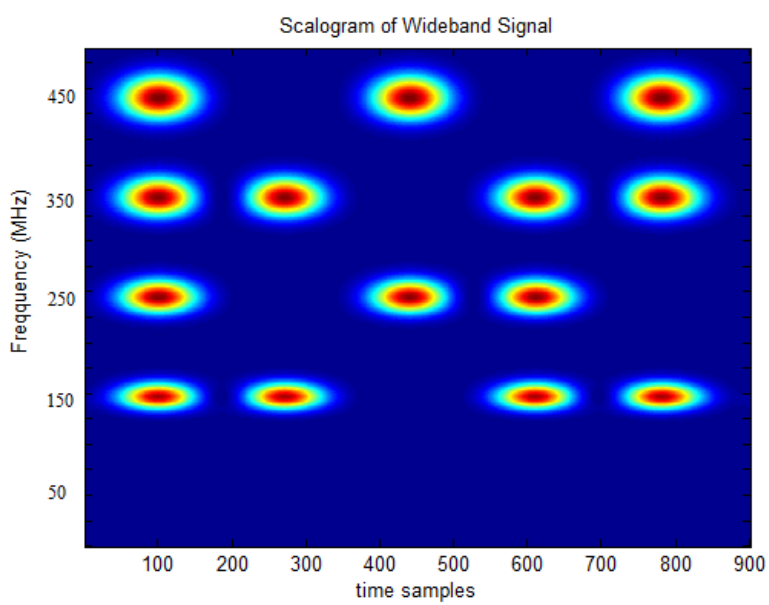

Fig. 4 Scalogram of the wideband signal with TFT

The power of each PU in joint time-frequency plane is illustrated in Fig. 5. It is depicted that the power of each PU is varied with time and frequency. From the PSD, it is also possible to identify the presence of PUs at every time instant.

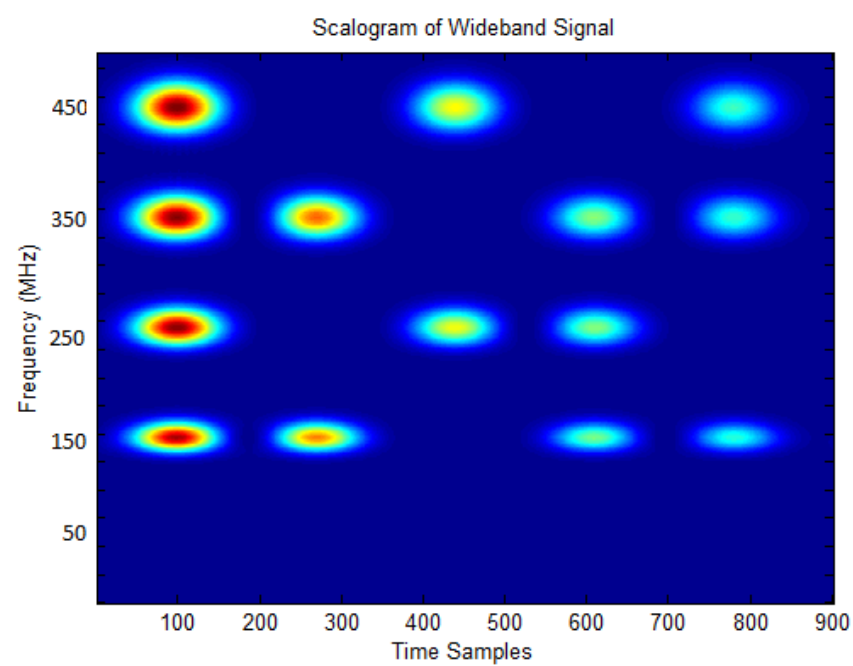

Fig. 5 Mesh diagram of the wideband signal with TFT The WSS with proposed MST under multipath fading channel are illustrated from Fig. 6 to Fig. 9. The sensing is done under fading channels such as Rayleigh and Rician.

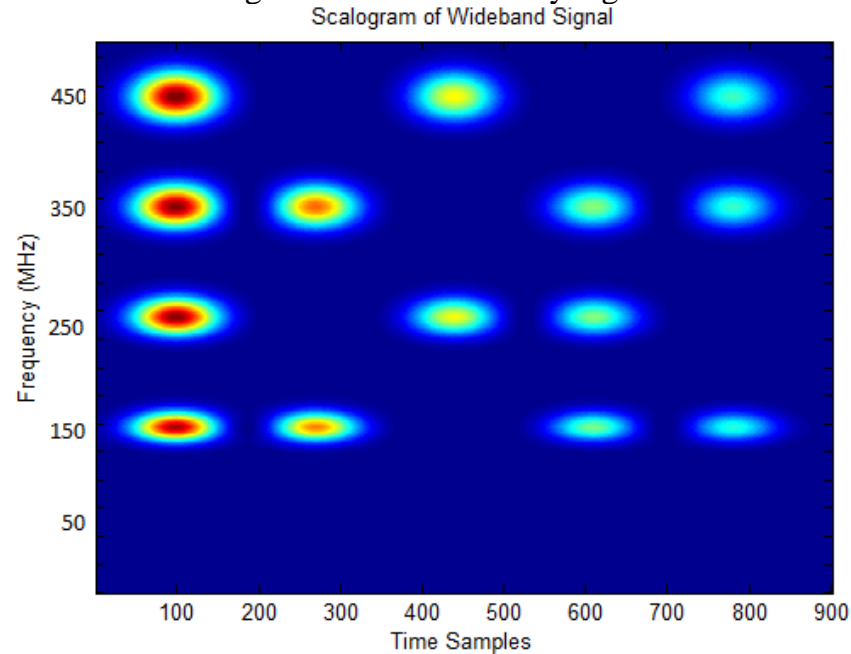

Fig. 6 WSS under Rician fading and AWGN with 10dB SNR

It is portrayed through the Fig. 6 that the wideband sensing under Rician fading channel using proposed TFT approach. For simulation, four paths are chosen with random path gains and doppler shifts. From the simulation result, it is observed that Rician fading doesn't affect the sensing accuracy even though the PSD of the PUs is changed with time.

It is depicted through the Fig. 7 that the wideband sensing is observed under Rayleigh fading channel using proposed TFT approach. For simulation, four paths are chosen with random path gains and doppler shifts. From the simulation result, it is examined that even though the received signal doesn't have any dominant component, it achieves better sensing accuracy. 


\section{Spectrum Sensing using AMC and TFT}

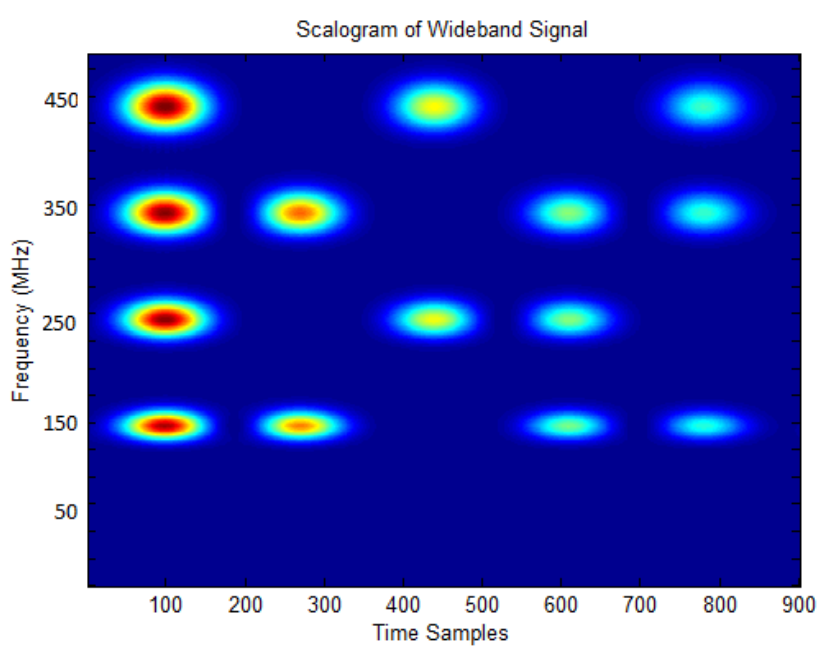

Fig. 7 WSS under Rayleigh fading and AWGN

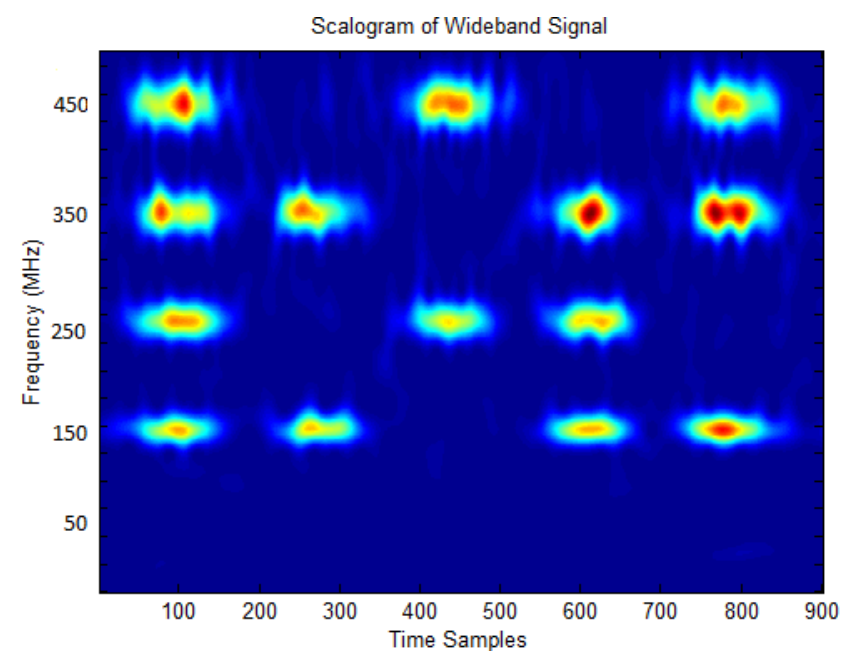

Fig. 8 WSS under Rician fading and AWGN

It is portrayed through the Fig. 8 that the wideband sensing using proposed TFT approach under Rician fading and AWGN is obtained with SNR of 0dB. From the simulation result, it is observed that the proposed method provides better performance under fading conditions, where most of the traditional techniques is failed in sensing.

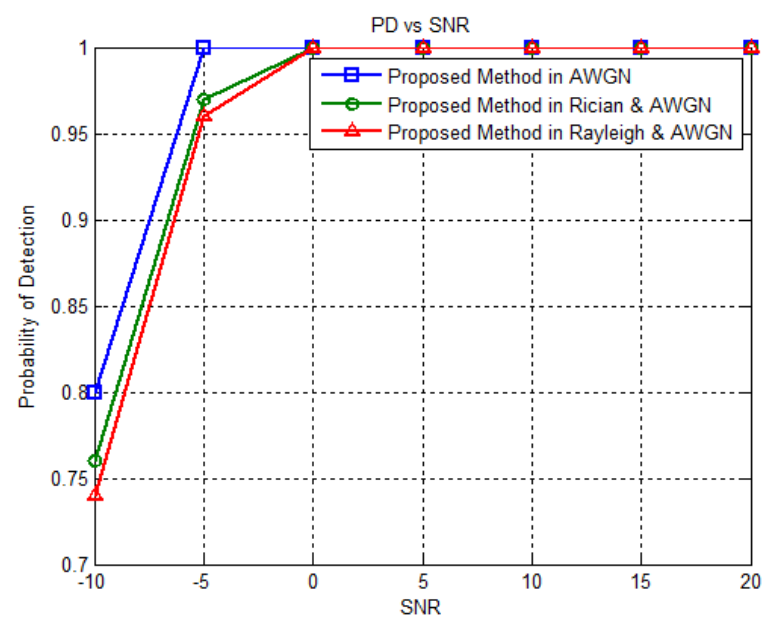

Fig. 9 Performance of under non-ideal channel conditions The performance comparison in terms of Probability of Detection (PD) of proposed approach under fading conditions is shown in Fig. 9. From this, it is depicted that proposed TFT based SS method provides more than $70 \%$ detection accuracy in AWGN and fading channel conditions even at $-10 d B$ SNR value.

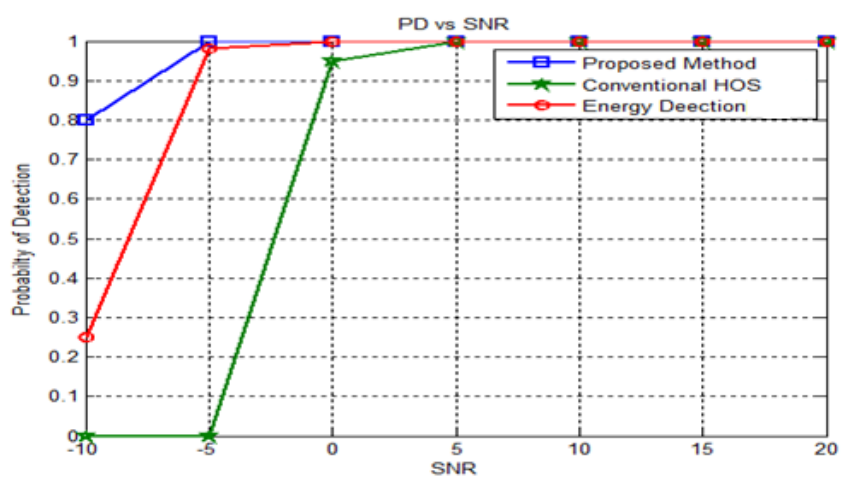

Fig. 10 Comparison of PD vs SNR in AWGN

The performance of proposed approach with that of existing methods is shown in Fig. 10. From this, it is depicted that at lower values of SNR, the proposed TFT based SS method provides better performance in terms of probability detection under AWGN channel conditions than the existing approaches.

\section{CONCLUSION}

A new algorithm is developed for narrowband and wideband SS using AMC and TFT. Spectrum scenario at any instant of time is visualized the proposed TFT\& AMC approach. Further, the PU and SU are distinguished with AMC algorithm. Different fading cases are considered to verify the efficiency of the proposed method for spectrum sensing. The performance of proposed AMC and TFT based spectrum sensing technique is analyzed under different channel conditions. From the analysis, it is proved that the TFT provides better performance for wideband spectrum sensing, compared to that of existing methods.

\section{REFERENCES}

1. S. Haykin, "Cognitive radio: Brain-empowered wireless communications," IEEE Journal on Selected Areas in Communications, vol. 23, no. 2, pp. 201-220, Feb. 2005.

2. T. Yucek and H. Arslan, "A survey of spectrum sensing algorithms for cognitive radio applications," IEEE Communications Surveys and Tutorials, vol. 11, no. 1, pp. 116-130, 2009.

3. H. Sun, A. Nallanathan, C.-X. Wang, and Y. Chen, "Wideband spectrum sensing for cognitive radio networks: A survey," IEEE Wireless Communications, vol. 20, no. 2, pp. 74-81, 2013.

4. A. Margoosian, J. Abouei, and K. N. Plataniotis, "An accurate kernelized energy detection in Gaussian and non-Gaussian/impulsive noises," IEEE Transactions on Signal Processing, vol. 63, no. 21, pp. 5621-5636, 2015.

5. C. Liu, M. Li, and M.-L. Jin, "Blind energy-based detection for spatial spectrum sensing," IEEE Wireless Communication Letters, vol. 4, no. 1, pp. 98-101, 2015

6. A. F. Eduardo and R. G. G. Caballero, "Experimental evaluation of performance for spectrum sensing: Matched filter vs energy detector," Proceedings of IEEE Colombian Conference on Communication and Computing (IEEE COLCOM 2015), Popayan, pp. 1-6, 2015.

7. A. Nasser, A. Mansour, K. C. Yao, H. Charara and M. Chaitou, "Efficient spectrum sensing approaches based on waveform detection," Proceedings of International Conference on e-Technologies and Networks for Development (ICeND2014), Beirut, pp. 13-17, 2014.

8. Narendar Madhavan, A.P. Vinod, A.S. Madhukumar, Anoop Kumar Krishna, "Spectrum sensing and modulation classification for cognitive radios using cumulants based on fractional lower order statistics," AEU International Journal of Electronics and Communications, vol. 67, no. 6, pp. 479-490, 2013.

Published By:

Blue Eyes Intelligence Engineering 
9. Z. Quan, S. Cui, A. H. Sayed, and H. V. Poor, "Optimal multiband joint detection for spectrum sensing in cognitive radio networks," IEEE Transactions on Signal Processing, vol. 57, no. 3, pp. 1128-1140, Mar. 2009.

10.M. Kim and J. Takada, "Efficient multi-channel wideband spectrum sensing technique using filter bank," Proceedings of IEEE 20th International Symposium on Personal, Indoor and Mobile Radio Communications, Tokyo, pp. 1014-1018, 2009.

11.Mengda Lin, A. P. Vinod and C. Samson, "Progressive decimation filter banks for variable resolution spectrum sensing in cognitive radios," Proceedings of 17th International Conference on Telecommunications, Doha, pp. 857-863, 2010.

12.Z. Tian and G. B. Giannakis, "A Wavelet Approach to Wideband Spectrum Sensing for Cognitive Radios," Proceedings of $1 \mathrm{st}$ International Conference on Cognitive Radio Oriented Wireless Networks and Communications, Mykonos Island, pp. 1-5, 2006.

13.M.Venkata Subbarao, P.Samundiswary "Time-Frequency Analysis of Non-Stationary Signals using Frequency Slice Wavelet Transform" IEEE International Conference on Intelligent Systems and Control (ISCO'16) Organized by department of IT, Karpagam College of Engineering, Coimbatore.

14.M. Venkata Subbarao, P. Samundiswary, "Spectrum Sensing in Cognitive Radio Networks Using Time-Frequency Analysis and Modulation Recognition", Springer Lecture Notes in Electrical Engineering, Vol 471, pp.827-837, Feb-2018.

15.M. Venkata Subbarao, P. Samundiswary, "Performance Analysis of Automatic Modulation Classification using Time Frequency Transforms under Non-Ideal Channel Conditions", International Journal of Innovative Technology and Exploring Engineering (IJITEE), Vol 8, Issue 12, pp.1685-1691, October 2019.

\section{AUTHORS PROFILE}

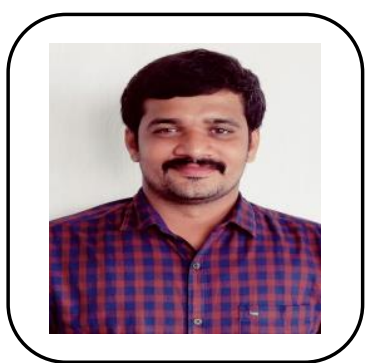

M. Venkata Subbarao received the B.Tech. degree in Electronics and Communication Engineering from Jawaharlal Nehru Technological University, Hyderabad, India in 2008 and Masters degree in Digital Electronics and Communication Systems from Jawaharlal Nehru Technological University, Kakinada, India, in 2011. He is currently doing his Ph.D. in Department of Electronics Engineering, as a part-time (External) scholar at Pondicherry University, Pondicherry, India. He has 9 years of teaching experience and presently he is working as Asst. Professor in the Dept. off ECE, Shri Vishnu Engineering College for Women, A.P. He has been selected for Visvesvaraya Fellowship, from the Department of Electronics and Information Technology, Ministry of Communication \& IT, Govt. of India for pursuing Ph.D. He has authored or co-authored over 35 technical publications. He received two "Best Paper Awards" for his technical papers, in Springer International Conference on "Micro-Electronics, Electromaganetics and Telecommunications" (ICMEET-2017) and International Conference on Communication and Security (ICCS 2016). He received "Best Teacher" award from the Tirumala Engineering College, A.P, India. He is a member of IE, IETE and IEEE. His current areas of research interests include Signal and Image Processing, Wireless Communications, Machine Learning and Pattern Recognition.

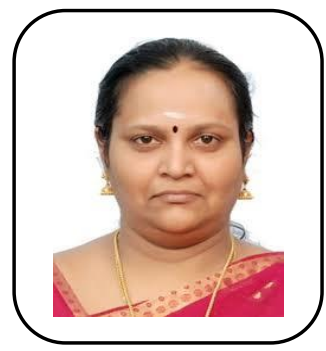

P. Samundiswary obtained her B.Tech degree and M.Tech. Degree in Electronics and Communication Engineering from Pondicherry Engineering College affiliated to Pondicherry Central University, Pondicherry, India in 1997 and 2003 respectively. She received her Ph.D. degree from Pondicherry Engineering College affiliated to Pondicherry Central University, Pondicherry, India in 2011. She has nearly 20 Years of teaching experience. Presently, she is working as Assistant Professor in the Department of Electronics Engineering, School of Engineering and Technology, Pondicherry Central University, India. She has published more than 100 papers in National and International conference proceedings and journals. She has been one of the authors of the book published by LAMBERT Academic Publishing, Germany. Also, she has co-authored five book chapters published by INTECH and Springer Publishers. Her areas of interest are Wireless Communication and Networks,
Computer Networks, Optical Communication and VLSI Design. She will be available at samundiswary_pdy@yahoo.com. 